\title{
Justification of an Asymptotic Expansion at Infinity
}

\author{
Leonid Kalyakin
}

Chenyshevski str. 112, Ufa, 450077, Russia

E-mail:klenru@mail.ru

\begin{abstract}
A family of asymptotic solutions at infinity for a system of ordinary differential equations is considered. Existence of exact solutions which have these asymptotics is proved.
\end{abstract}

\section{Introduction}

This paper is a reply to the Calogero's question, asked on the NEEDS-2007, about justification of asymptotic solution of ordinary differential equations. The full-dimensional manifold of solutions is the principal interest. Such solutions are usually linked with the physically observed phenomena. In the case of linear equations an asymptotic expansion of the general solution can be constructed by using a fundamental system of asymptotic solutions $[5,15]$. We present here a similar result for nonlinear equations.

The main object is the system of differential equations

$$
\frac{d \mathbf{x}}{d t}=t^{k} \mathbf{f}(\mathbf{x}, t), \quad \mathbf{x} \in \mathbb{R}^{n},(k=\text { const } \geq 0)
$$

considered at $t \geq t_{0}>0$. The factor $t^{k}$ is here involved for identification of growing right hand sides, occurring in applied problems; $k+1$ is called Poincare rank. Let $\mathbf{X}(t ; \alpha), \alpha \in \mathbb{R}^{n}$ be an $n$-parametric asymptotic solution at infinity. It means that the vector function $\mathbf{X}(t ; \alpha) \in C^{1}\left[t_{0}, \infty\right)$ under substitution into the equation (1.1) yields the residual

$$
\mathbf{Y}(t ; \alpha) \equiv \frac{d \mathbf{X}}{d t}-t^{k} \mathbf{f}(\mathbf{X}, t),
$$

which is vanishing at infinity: $\mathbf{Y}(t ; \alpha)=O\left(t^{-\mu}\right), t \rightarrow \infty, \mu=$ const $>0$. We study the following problem: is there $n$-parametric exact solution $\mathbf{x}(t ; \alpha)$, for which the $\mathbf{X}(t ; \alpha)$ is an asymptotic approximation at infinity: $\mathbf{x}(t ; \alpha)=\mathbf{X}(t ; \alpha)+O\left(t^{-v}\right), t \rightarrow \infty,(v=$ const $>0)$ ? An affirmative answer is obtained here. The proof is reduced to the existence theorem for the remainder. The principal feature is the uniformity of the asymptotics with respect to the parameter $\alpha$.

Justification of asymptotics in the general case was not proved up to now and, probably, can not be proved at all, although very interesting results are known for some types of equations. Results for linear equations are collected in $[5,15]$. Justification of asymptotics for the nonlinear Painlevé equations are discussed in $[2,7,9]$. Usually the existence theorem follows the formal 
asymptotic construction. Various theorems were proved under different conditions which depend on the asymptotic methods used [3, 12, 11, 13, 14]. Kuznetsov's well known results [13, 14] deal with the case when $X(t)$ is a formal series (of powers, logarithms and exponentials) with constant coefficients. Sometimes such series give an asymptotic expansion of the general solution [11]. But often such an expansion does not correspond to general solution. For example, the pendulum equation $\ddot{x}+\sin x=0$ has few solutions of that type: a single solution $x(t) \equiv 0$ and two one-parametic solutions $x(t)= \pm \pi+O(\exp (-t)), t \rightarrow \infty$. The other solutions are oscillating and belong to a two-parametric family.

Usually the general asymptotic expansions for nonlinear equations are given by WKB formulas in the form of power series with oscillating coefficients. P.Boutroux studied such two-parametric asymptotics for the Painlevé equations [2]. In the general case the WKB asymptotics depend on $n$ parameters and just such solutions are of interest in physics. For example, existence of twoparametric increasing solution of main resonance equations is identified with the presence of the autoresonance phenomenon $[4,10]$. Justification of WKB asymptotics for the linear equations $[5,16]$ and for some nonlinear equations [11] can be reduced to Kuznetsov's theorem. But the reduction is unknown and evidently is impossible for many equations occurred in physics. Painleveś equations studied in $[2,7,9]$ give examples of such type.

Justification of WKB asymptotics for the Painlevé equations is usually based on the property of integrability [7]. Nonlinear nonintegrable equations are studied in this paper. We prove an existence theorem and give an estimate for the remainder. The presence of the $n$-parameter solution makes the proof very easy. Both technic of the proof and the derived estimate are determined by the properties of the asymptotic solution $\mathbf{X}(t ; \alpha)$. A similar estimate obtained in [8] for the case of single solution $\mathbf{X}(t)$ is linked with the properties of matrix of linearized system $\partial_{\mathbf{X}} \mathbf{f}(\mathbf{X}, t)$. Both of these results are obtained under different sufficient conditions, hence in general case they can not be reduced to each other. In order to perform a comparison one has to specify the considered system and the asymptotic solution. We avoid both any asymptotic construction and any discussion on the method which yields the asymptotic solution. This approach allows us to obtain an existence theorem in general form which may be useful for a wide range of problems.

Our estimate is uniform with respect to the parameter $\alpha$ and this feature is crucial for the successful application in asymptotic theories. In particular the uniformity of the WKB asymptotics proves the orbital stability of the oscillating solutions. Researches $[13,14,8]$ deal with a single solution which may be stable or unstable, see [11]. As a rule the solution have no any physical sense, if it is unstable. Stability of the solution is not discussed in the existence theorems $[13,14$, 8]. The problem of stability returns us to investigation of $n$-parameter solution which is considered here. Certainly our result does not give any information on unstable solutions.

\section{Input conditions}

We consider the equation (1.1) under the assumption that the vector function from the right side $\mathbf{f}(\mathbf{x}, t)$ is smooth (continuously differentiable) in the domain $\left\{\mathbf{x} \in D \subseteq \mathbb{R}^{n}, t \geq t_{0}\right\}$. An asymptotic behavior at infinity

$$
\mathbf{f}(\mathbf{x}, t), \frac{\partial \mathbf{f}(\mathbf{x}, t)}{\partial \mathbf{x}}=O(1), \quad t \rightarrow \infty
$$

uniformly on every compact $\mathbf{x} \in \mathscr{K} \subset D$ is supposed. The principal conditions are formulated by means of the asymptotic solution $\mathbf{X}(t ; \alpha)$. It is assumed that the vector function $\mathbf{X}(t ; \alpha)$ depends 
on the parameters $\alpha=\left(\alpha_{1}, \ldots, \alpha_{n}\right)$ and this dependence is smooth in some domain $\alpha \in A_{0} \subseteq$ $\mathbb{R}^{n}$. Values of the asymptotic solution belong to the compact ${ }^{1}: \mathbf{X}(t ; \alpha) \in \mathscr{K}_{\mathscr{A}} \subset \mathbb{R}^{n}$ when both $t \in\left[t_{0}, \infty\right)$ and parameters $\alpha$ range over any compact: $\alpha \in \mathscr{A} \subset A_{0}$. Parameters $\alpha$ are supposed functionally independent and the Jacobian

$$
\mathscr{J}(t ; \alpha) \equiv \frac{\partial \mathbf{X}}{\partial \alpha}
$$

has an asymptotics

$$
\operatorname{det} \mathscr{J}(t ; \alpha)=t^{p}[a+o(1)], t \rightarrow \infty,(p=\text { const, } a=\text { const } \neq 0) \text {. }
$$

In some sense $\mathbf{X}(t ; \alpha)$ is a general asymptotic solution. Elements of the Jacobian matrix $J=\left\{a_{i, j}\right\}$ have a similar asymptotics (may be different order for various number $i, j=1, \ldots, n$ )

$$
a_{i, j}(t ; \alpha)=O\left(t^{q_{i, j}}\right), t \rightarrow \infty,\left(q_{i, j}=\mathrm{const}\right) .
$$

We shall use the more short formula which may be considered as an asymptotic estimate:

$$
\mathscr{J}(t ; \alpha)=O\left(t^{q}\right), t \rightarrow \infty,\left(q=\max q_{i, j}\right) .
$$

Under these assumptions the inverse matrix has a similar asymptotic estimate

$$
\mathscr{J}^{-1}(t ; \alpha)=O\left(t^{r}\right), t \rightarrow \infty .
$$

One can see that the exponents satisfy the relations: $p \leq q n, r \leq q(n-1)-p$.

The residual determined by the formula (1.2) depends on the parameters as well: $\mathbf{Y}=\mathbf{Y}(t ; \alpha)$. We assume asymptotic estimates:

$$
\mathbf{Y}(t ; \alpha)=O\left(t^{-\mu}\right), \frac{\partial \mathbf{Y}}{\partial \alpha}=O\left(t^{-\mu+s}\right), t \rightarrow \infty,(\mu, s=\text { const }) .
$$

All asymptotics given above are supposed to be uniform with respect to parameters $\alpha \in \mathscr{A}$.

Remark. All restrictions are induced by examples of WKB asymptotics for the Painlevé's equations $[2,7,9]$ and for the main resonance equations [10]. The exponent $\mu$ is a crucial parameter. It corresponds to the length of the asymptotic solution. As a rule, $\mu$ can be made large as desired $^{2}$, while the exponents $k, p, q, r, s$ depend on both the considered equations and the family of asymptotic solutions. They do not depend on the length of asymptotics $\mu$, as is seen in different examples.

\section{Existence theorem}

Theorem 1. Let the vector function $\mathbf{X}(t ; \alpha), \alpha \in A_{0} \subseteq \mathbb{R}^{n}$ be an n-parametric asymptotic solution of the equation (1.1) and the properties (2.1), (2.2) hold. If the residual defined by (1.2) is decreased (2.3) fast sufficiently at infinity:

$$
\mu>r+s+1 \text { and } \mu>2(r+q+1)+k,
$$

\footnotetext{
${ }^{1}$ The case of unbounded asymptotic solution can be reduced to considered one.

${ }^{2}$ In the case of complicated problem the quantity $\mu$ depends on the talent of researcher, [1].
} 
then for arbitrary compact $\mathscr{A} \subset A_{0}$ there is a neighborhood of infinity $t \in[T, \infty)$ where the equation (1.1) has the n-parametric exact solution $\mathbf{x}(t ; \alpha)$ which has an asymptotics

$$
\mathbf{x}(t ; \alpha)=\mathbf{X}(t ; \alpha)+O\left(t^{-v}\right), t \rightarrow \infty,(v=\mu-r-q-1>0)
$$

uniformly with $\forall \alpha \in \mathscr{A}$.

Proof. The idea of the proof was suggested by M.Fedoruk [6] and later was applied in [10].

We seek an exact solution as the sum $\mathbf{x}(t ; \alpha)=\mathbf{X}(t ; \alpha)+\mathbf{R}(t ; \alpha)$. The dependence on the parameters $\alpha$ will be omitted for brevity. The equation for the desired remainder $\mathbf{R}$ reads

$$
\frac{d \mathbf{R}}{d t}=t^{k}[\mathbf{f}(\mathbf{X}+\mathbf{R}, t)-\mathbf{f}(\mathbf{X}, t)]-\mathbf{Y}(t) .
$$

It can be rearranged by identification of the linear part of the operator as follows

$$
\frac{d \mathbf{R}}{d t}=t^{k}\left[\mathscr{M}_{0}(t) \mathbf{R}+\mathbf{G}(\mathbf{R}, t)\right]-\mathbf{Y}(t) .
$$

Here the matrix $\mathscr{M}_{0}(t)$ is calculated by means of derivatives of the known functions given above:

$$
\mathscr{M}_{0}=\frac{\partial \mathbf{f}(\mathbf{X}, t)}{\partial \mathbf{x}} \text {. }
$$

The vector function

$$
\mathbf{G}(\mathbf{R}, t)=\mathbf{f}(\mathbf{X}+\mathbf{R}, t)-\mathbf{f}(\mathbf{X}, t)-\partial_{\mathbf{X}} \mathbf{f}(\mathbf{X}, t) \mathbf{R}
$$

has an asymptotics $\mathbf{G}(\mathbf{R}, t)=O\left(|\mathbf{R}|^{2}\right)$ as $\mathbf{R} \rightarrow 0$. This asymptotics is uniform with respect to $t \geq t_{0}$.

We desire to demonstrate existence of an exact solution $\mathbf{R}(t ; \alpha)$ which is given in some infinite interval $T \leq t<\infty$ and which is rapidly decreasing at infinity. As usual in such theorems, the differential equation (3.3) must be reduced to an integral equation in order to apply the method of successive approximations. However, a trivial reduction consisting in integration with respect to $t$ does not lead to success because the linear part of the integral operator is not contractive in that case. The idea suggested by M.Fedoruk consists in inversion of an essential part of the linear operator in the equation (3.3) by using the given asymptotic solution $\mathbf{X}(t ; \alpha)$. For this type of inversion we use the Jacobi matrix $\mathscr{J}(t ; \alpha)$. It is a fundamental asymptotic solution for the linearized equation. Indeed, derivation of the starting identity (1.2) with respect to parameters $\alpha$ yields

$$
\frac{d \mathscr{J}}{d t}-t^{k} \mathscr{M}_{0}(t) \mathscr{J}=\partial_{\alpha} \mathbf{Y} .
$$

Here the left hand side represents linearized operator which is applied at $J$, while the right hand side is the residual which is rapidly vanishing at infinity: $\partial_{\alpha} \mathbf{Y}(t ; \alpha)=O\left(t^{s-\mu}\right), t \rightarrow \infty$.

The Jacobi matrix is used in the substitution $\mathbf{R}=\mathscr{J} \mathbf{C}$. Then the equation (3.3) is transformed at the equation for the vector function $\mathbf{C}(t ; \alpha)$ :

$$
\frac{d \mathbf{C}}{d t}=\mathscr{M}(t) \mathbf{C}+\mathscr{G}(\mathbf{C}, t)-\mathscr{J}^{-1} \mathbf{Y}(t) .
$$

The main novelty now is the matrix $\mathscr{M}$ in the linear part

$$
\mathscr{M}(t)=-\mathscr{J}^{-1} \partial_{\alpha} \mathbf{Y}=O\left(t^{r+s-\mu}\right),
$$


which is rapidly decreasing at infinity if the $\mu$ is sufficiently large. In particular, the norm of the matrix is estimated in $\mathbb{R}^{n}$

$$
|\mathscr{M}(t)| \leq M_{0} t^{r+s-\mu}, \forall t \geq t_{0},\left(M_{0}=\mathrm{const}\right) .
$$

The nonlinear part is represented by the vector function

$$
\mathscr{G}(\mathbf{C}, t)=t^{k} \mathscr{J}^{-1} \mathbf{G}(\mathscr{J} \mathbf{C}, t) .
$$

It has an asymptotics $\mathbf{G}(\mathbf{R}, t)=O\left(|\mathbf{R}|^{2}\right)$ as $|\mathbf{R}| \rightarrow 0$ and this asymptotics is differentiable with respect to $\mathbf{R}$ (uniformly with respect to $t$ ). Then the inequality

$$
\left|\mathbf{G}\left(\mathbf{R}_{1}, t\right)-\mathbf{G}\left(\mathbf{R}_{2}, t\right)\right| \leq M_{K}\left(\left|\mathbf{R}_{1}\right|+\left|\mathbf{R}_{2}\right|\right)\left|\mathbf{R}_{1}-\mathbf{R}_{2}\right|, \quad \forall t \geq t_{0}
$$

holds for all $\mathbf{R}_{1}, \mathbf{R}_{2}$ in arbitrary compact $\mathscr{K}$; here the constant $M_{K}$ depends on the chosen compact $\mathscr{K}$. Last relation provides the Lipschitzian property of the operator:

$$
\left|\mathscr{G}\left(\mathbf{C}_{1}, t\right)-\mathscr{G}\left(\mathbf{C}_{2}, t\right)\right| \leq M_{K} t^{k+r} \cdot t^{q}\left(\left|\mathbf{C}_{1}\right|+\left|\mathbf{C}_{2}\right|\right) \cdot t^{q}\left|\mathbf{C}_{1}-\mathbf{C}_{2}\right|
$$

for all vector function $t^{q} \mathbf{C}_{1}(t), t^{q} \mathbf{C}_{2}(t)$ in every compact $\mathscr{K}$.

The differential equation (3.4) is equivalent to the integral equation

$$
\mathbf{C}(t)-\int_{\infty}^{t}[\mathscr{M}(\eta) \mathbf{C}(\eta)+\mathscr{G}(\mathbf{C}(\eta), \eta)] d \eta=\mathbf{Z}(t) .
$$

The vector function on the right hand side

$$
\mathbf{Z}(t)=\int_{t}^{\infty} \mathscr{J}^{-1} \mathbf{Y}(\eta) d \eta
$$

is well known and has an asymptotics $\mathbf{Z}(t)=O\left(t^{r+1-\mu}\right), t \rightarrow \infty$. We are looking for a solution $\mathbf{C}(t)$ which has a like asymptotic behavior.

In order to prove the theorem, we have to introduce some Banach space. An appropriate space of continuous vector functions $\mathbf{C}(t) \in C_{\lambda}[T, \infty)$ is determined by the weight norm

$$
\|\mathbf{C}\|_{\lambda}=\sup _{t \geq T} t^{\lambda}|\mathbf{C}(t)|, \lambda=\mu-r-1 .
$$

The boundary $T=$ const $\geq t_{0}$ will be chosen by the requirement of contractiveness of the integral operator in what follows.

The first condition from (3.1) taken in the form $\lambda>q$ and the inequality (3.5) provide the estimate

$$
\left|\mathscr{G}\left(\mathbf{C}_{1}, t\right)-\mathscr{G}\left(\mathbf{C}_{2}, t\right)\right| \leq M_{K} t^{k+r+2 q-2 \lambda} \cdot\left(\left\|\mathbf{C}_{1}\right\|_{\lambda}+\left\|\mathbf{C}_{2}\right\|_{\lambda}\right)\left\|\mathbf{C}_{1}-\mathbf{C}_{2}\right\|_{\lambda}
$$

which is valid for all vector functions $\mathbf{C}_{1}(t), \mathbf{C}_{2}(t)$ in arbitrary compact $\mathscr{K} \subset C_{\lambda}[T, \infty)$. If we take here the integral with $t$, then we can derive the Lipschitzian inequality

$$
\left\|\int_{\infty}^{t}\left[\mathscr{G}\left(\mathbf{C}_{1}, \eta\right)-\mathscr{G}\left(\mathbf{C}_{2}, \eta\right)\right] d \eta\right\|_{\lambda} \leq M_{K} 2 K T^{k+r+2 q+1-\lambda} \cdot\left\|\mathbf{C}_{1}-\mathbf{C}_{2}\right\|_{\lambda} \equiv L_{K} \cdot\left\|\mathbf{C}_{1}-\mathbf{C}_{2}\right\|_{\lambda},
$$


which is valid for all $\left\|\mathbf{C}_{1}\right\|_{\lambda},\left\|\mathbf{C}_{2}\right\|_{\lambda} \leq K$. The Lipschitzian constant

$$
L_{K}=M_{K} 2 K T^{k+2(r+q+1)-\mu}
$$

can be chosen arbitrary small (for example, $L_{K}<1 / 2$ ) under arbitrarily large $T$, as the exponent $k+2(r+q+1)-\mu<0$ is negative because of the second condition (3.1). The relation (3.8) provides contractiveness of the nonlinear part of the integral operator.

The norm of the linear part of the integral operator can be estimated by integration of the matrix norm $\mathscr{M}(t)$ :

$$
\begin{aligned}
& \sup _{t \geq T} t^{\lambda} \int_{t}^{\infty} \eta^{-\lambda}|\mathscr{M}(\eta)| d \eta \leq M_{1} \sup _{t \geq T} t^{\lambda} \int_{t}^{\infty} \eta^{r+s-\mu-\lambda} d t= \\
& =M_{1} T^{r+s+1-\mu} /(r+s-\mu-\lambda+1) \equiv L_{0} .
\end{aligned}
$$

The constant $L_{0}$ can be made small as desired (say, $L_{0}<1 / 2$ ) under assumed condition $\mu>$ $r+s+1$. It is enough to choose an appropriate large $T$. The $L_{0}$ is used as a Lipschitzian constant for the linear part of integral operator:

$$
\left\|\int_{\infty}^{t} \mathscr{M}(\eta)\left[\mathbf{C}_{1}(\eta)-\mathbf{C}_{1}(\eta)\right] d \eta\right\|_{\lambda} \leq L_{0}\left\|\mathbf{C}_{1}-\mathbf{C}_{2}\right\|_{\lambda}
$$

Thus, if the value of $T$ is sufficiently large then the integral operator from the equation (3.6) turn out to be contractive in Banach space $C_{\lambda}[T, \infty)$. The right side of the equation $\mathbf{Z}(t)=$ $O\left(t^{r+1-\mu}\right), t \rightarrow \infty$ belongs the same space $\mathbf{Z}(t) \in C_{\lambda}[T, \infty)$ as $\lambda=\mu-r-1$. Then the fixedpoint theorem can be applied to the integral equation (3.6). It implies existence of the solution $\mathbf{C}(t) \in C_{\lambda}[T, \infty)$. This vector function is differentiable because of the identity (3.4). As the equation (3.4) is equivalent to (3.2), then $\mathbf{R}(t)=\mathscr{J} \mathbf{C}(t)$ is a desired solution of the residual equation (3.2) and it has an asymptotics

$$
\mathbf{R}(t)=\mathscr{J} \mathbf{C}(t)=O\left(t^{q-\lambda}\right), t \rightarrow \infty,(\lambda-q=v) .
$$

It must be point out that all estimates found are uniform with respect to parameters $\alpha$ in arbitrary compact $\mathscr{A}$. So the proof, as given above, is suitable both for the case of a fixed parameter $\alpha$ and a fixed compact: $\alpha \in \mathscr{A} \subset A_{0}$. In the last case the estimate of the remainder

$$
\mathbf{R}(t ; \alpha)=\mathscr{J} \mathbf{C}(t ; \alpha)=O\left(t^{-v}\right), t \rightarrow \infty
$$

is uniform with respect to $\forall \alpha \in \mathscr{A}$. This completes the proof.

Remark. It may occur in application to specific problems that the order of the asymptotic estimate of the remainder $\mathbf{R}(t)=O\left(t^{\mu-r-q-1}\right)$ is very rough. More precise estimations can be derived, if a full asymptotic solution in the form of an infinite asymptotic series is known; see [10].

Acknowledgments. The research was supported by RFBR grants nos. 06-01-00124, 06-0192052, INTAS grant no. 03-51-4286 and grant of Scientific School no. 2215.2008.1. 


\section{References}

[1] BABICH V M and BuldyreV V S, Art of asymptoics, Vestnik LSU, 13 (1977), 5-12. (in Russian)

[2] Boutroux P, Recherches sur les transcendantes de M.Painlevé et l'étude asymptotique des équations differéntielles du second ordre, Ann. Sci. Ec. Norm. Super. 30, (1913), 265-375; 31, (1914), 99-159.

[3] BRuno A D, Power geometry in algebraic and differential equations. Moscow, Nauka, (1998). (in Russian)

[4] FAJAns J AND FRIEDland L Autoresonant (non stationary) excitation of a pendulum, Plutinos, plasmas and other nonlinear oscillators, Am. J. Phys. 69, (2001), 1096-1102.

[5] Fedoruk M V, Asymptotic methods for ordinary differential equations. Nauka, Moscow, 1983. (in Russian)

[6] FedoruK M V, WKB method for nonlinear second order equation, Journ vychisl. math. and math. phys. 26, (1986), 196-210. (in Russian)

[7] Fokas A S, Its A R, Kapaev A A, and Novokshenov V Yu, Painlevé Transcendents: The Riemann-Hilbert Approach, AMS, 2006.

[8] Gingold H, Tovbis A, Solution of finitely smooth nonlinear differential equations and problems of diagonalization and triangularization, SIAM J. Math. Anal. 29, (1998), 757-778.

[9] Joshi N, Asymptotic Studies of the Painlevé Equations / The Painlevé Property. One Centure Later. CRM Series in Math. Phys. Springer. New York. (1999), 181-228.

[10] Kalyakin L A, Justification of Asymptotic Expansions for the Principal Resonance Equations, Proc. of the Steklov Inst. of Math. Suppl. 1, (2003), S108-S122.

[11] KOZLOV V V and FurTa S D, Asymptotics of solutions of strong nonlinear systems of differential equations. MSU, Moscow, 1996. (in Russian)

[12] KudryavtceV L D, Asymptotics of solutions of differential equations in the neighborhood of singular points, Proc. of the Steklov Inst. of Math. 232, (2001), 194-217. (in Russian)

[13] Kuznetsov A N, Differentiable solutions of degenerated systems of ordinary equations, Funk. analis and his appl. 6, (1972), 41-51. (in Russian)

[14] KuZNETSOV A N, On existence of solutions entering in singular point of autonomous system which have a formal solution, Funk. analis and his appl. 23, (1989), 63-74. (in Russian)

[15] Olver F W J, Asymptotics and special functions, Academic Press, New York, 1974.

[16] Wasow W A, Asymptotic expansions for ordinary differential equations, Wiley, New York, 1965. 